\title{
Delayed Thalamic Intracranial Hemorrhage in an Essential Tremor Patient following Deep Brain Stimulation
}

\author{
Theresa A. Zesiewicz Kelly L. Sullivan Michael Hoffmann Lourdes M. Benes \\ Donald A. Smith Christopher L. Ward Robert A. Hauser
}

Parkinson's Disease and Movement Disorders Center, Department of Neurology, Department of Molecular Therapeutics, Neuroscience Program, National Parkinson's Foundation Center of Excellence, Department of Psychiatry, Department of Neurosurgery, University of South Florida, Tampa, Fla., USA

\begin{abstract}
Dear Sir,
Essential tremor (ET) is a common movement disorder characterized by an action or postural tremor [1] that is often difficult to treat pharmacologically. Patients may not respond to medications, or may experience side effects that limit their use [2]. Deep brain stimulation (DBS) is an effective surgical treatment for ET that is refractory to medical management. The procedure uses high-frequency electrical stimulation from an implanted electrode to modify activity of the ventral intermediate (VIM) nucleus of the thalamus. We report the case of a man with ET and a history of controlled hypertension who developed a delayed thalamic intracerebral hemorrhage (ICH) almost 9 weeks following DBS surgery.
\end{abstract}

\section{Case Report}

The patient was a 70 -year-old righthanded man who was diagnosed with ET 10 years ago, and who presented to a Veterans Administration movement disorders clinic for treatment of his tremor. The tremor affected both upper extremities, and greatly interfered with writing, pouring liquids, eating, and drinking. He was taking primidone $100 \mathrm{mg}$ twice daily, and could not tolerate higher doses due to somnolence. The patient had also taken topiramate (50 mg twice daily) and propranolol (40 mg twice daily) in the past for tremor control, but discontinued both medications several years ago due to side effects (paresthesias with topiramate and dizziness with propranolol). Family history included ET in the patient's mother and brother. Past medical history included hypertension, coronary artery disease, coronary artery 4 -vessel bypass graft (CABG) 4 years ago, and hyperlipidemia. The patient's hypertension had been well controlled for the 6 months before evaluation. He quit smoking 30 years ago, and currently drank 1-2 alcohol drinks per week. His medications at the time of assessment included one aspirin per day (81 mg) prescribed after the CABG, felodipine (Plendil) $2.5 \mathrm{mg}$ once daily, metoprolol (Toprol) $25 \mathrm{mg}$ once daily, iron sulfate 325 mg three times a day, fosinopril (Monopril) $40 \mathrm{mg}$ per week, hydrochlorothiazide (Vaseretic) $12.5 \mathrm{mg}$ once a day, simvastatin (Zocor) $20 \mathrm{mg}$ once daily, melatonin $3 \mathrm{mg}$ once a day at nighttime for sleep, omeprazole (Prilosec) $20 \mathrm{mg}$ twice a day, and primidone (Mysoline) $100 \mathrm{mg}$ twice a day. The patient's general physical examination, vital signs, and neurological exam were normal except for a mild head and voice tremor, and severe kinetic and postural tremor in both upper extremities. Fahn Tolosa Marin clinical rating scale [1] scores were 12 for Part A, 26 for Part B, and 19 for Part C. For the right side only, the score for Part A was 9 and for Part B it was 20. Laboratory tests including complete blood count, serum chemistries, and coagulant profile were normal, as was magnetic resonance imaging (MRI) of the brain.

The patient opted to receive unilateral DBS into the left VIM nucleus of the thalamus to control severe right upper extremity tremor. Aspirin was stopped 1 week before the surgery. He underwent stereotactic implantation of DBS electrodes (Model 3387; Medtronic, Minneapolis, Minn., USA) into the left VIM nucleus of the thalamus using microelectrode recording (MER) and 3 electrode passes. Electrodes were connected to a stimulator (Soletra, Medtronic) that was implanted in the subclavian area. Vital signs were normal during surgery, and there were no perioperative complications. After 4 weeks, stimulation parameters were turned on for the first time, for optimum tremor control, with complete resolution of the right upper extremity tremor. The patient resumed

\section{KARGER}

Fax +41 613061234

E-Mail karger@karger.ch

www.karger.com (c) 2008 S. Karger AG, Basel

0014-3022/08/0594-0187\$24.50/0

Accessible online at:

www.karger.com/ene
Theresa A. Zesiewicz, MD, FAAN

University of South Florida

12901 Bruce B. Downs Blvd, MDC Box 55

Tampa, FL 33612 (USA)

Tel. +1 813974 5909, Fax +1 813974 8032, E-Mail tzesiewi@health.usf.edu 

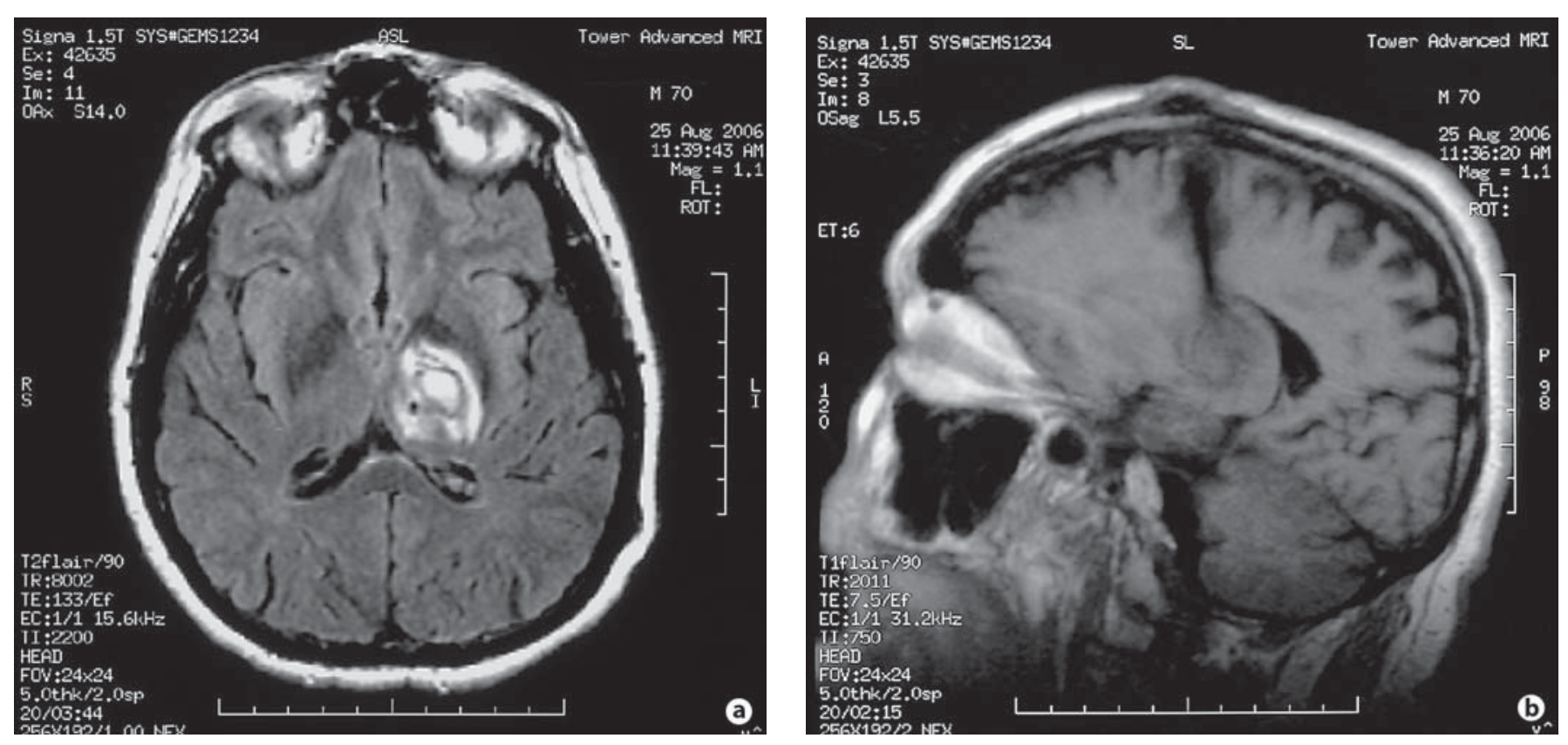

Fig. 1. Axial (a) and sagittal (b) views of MRI of the brain show left-sided thalamic ICH adjoining the distal portion of the deep brain stimulator of the electrode nearly 9 weeks after implantation of DBS.

taking one aspirin $(81 \mathrm{mg})$ daily $61 / 2$ weeks after surgery.

The patient returned to the movement disorders specialist (T.A.Z.) almost 9 weeks after DBS surgery for a routine follow-up, and his blood pressure, vital signs, and physical examination were normal. There was no evidence of tremor in the right upper extremity, and Fahn Tolosa Marin scores improved to 8 for Part A (4 for right side only), 8 for Part B (2 for right side only), and 7 for Part C. An MRI of the brain and a computed tomography (CT) of the brain taken postoperatively on the day of the surgery were normal. The day after this evaluation, the patient woke up from an afternoon nap with right-sided arm and leg weakness, slurred speech, and a right facial droop. The patient's wife recalls that he took all of his medication that morning as prescribed. She called emergency services, and the patient was transported to a hospital. One blood pressure reading in the ambulance was $170 / 85$, but all other pressures taken before and after this reading were normal. In the ER, vital signs were stable, and general physical examination was normal. Neurologically, the patient was lethargic, oriented to his name and disoriented to place and time. Cranial nerve examination was significant for a right facial paresis. Motor examination demonstrated right upper and lower extremity weakness (3/5). Sensory examination demonstrated right hemihypoesthesia, and the patient had an extensor plantar reflex on the right. Serum chemistries and complete blood count, platelets, prothrombin time, thyroid function tests, partial thromboplastin time (PTT), and sedimentation rate were normal, although triglycerides were elevated at $287 \mathrm{mg} / \mathrm{dl}$ (normal $\leq 150 \mathrm{mg} / \mathrm{dl}$ ), homocysteine was increased at $12.6 \mu \mathrm{M}($ normal $<10 \mu \mathrm{M})$ and low-density lipoprotein was decreased at $56 \mathrm{mg} / \mathrm{dl}$ (normal $\leq 100 \mathrm{mg} / \mathrm{dl}$ ). The electrocardiogram showed normal sinus rhythm with a heart rate of 60 beats per minute. CT and MRI of the brain without contrast showed a left-sided thalamic ICH adjoining the distal portion of the deep brain stimulator electrode, measuring $1.3 \times 1.8 \mathrm{~cm}$ with an estimated volume equal to $3 \mathrm{~cm}^{3}$, and tracking along the path of the electrode into the left frontal lobe. There was peripheral vasogenic edema with mild mass effect on the left lateral ventricle from the bleed (fig. 1). The patient was transferred to a neurologic intensive care unit, where he received supportive care. The aspirin was stopped and the DBS device was turned off. His blood pressures remained stable, and he improved clinically having normal MMSE, mild dysarthria, mild right facial weakness, and right upper and lower extremity weakness (4/5) in the ensuing 6 weeks. He was able to ambulate with the use of a walker.

\section{Discussion}

This is the case of a delayed thalamic ICH almost 9 weeks following DBS surgery into the VIM nucleus of the thalamus for tremor control. ICH is a potential adverse event of DBS stimulation of the VIM nucleus of the thalamus for ET and the subthalamic thalamic nucleus (STN) in Parkinson's disease. Perioperative intracranial hemorrhage is estimated to occur in $1-4 \%$ of patients [2]. One 6 -year retrospective evaluation of ICH found that thalamic DBS is significantly less likely to result in ICH compared to STN DBS $(1.2 \%$ risk with thalamic DBS vs. $2.2 \%$ risk with STN DBS, $p=0.001$ ) [3]. Previous cases of ICH following DBS that have been reported in the literature have only occurred perioperatively.

While the risk of hematoma formation in stereotactic procedures ranges from 1 to $4 \%$, one prospective analysis of 361 proce- 
dures performed by one surgeon found that the risk of bleeding for morphological procedures (biopsies, abscess evacuation, cyst evacuation, hematoma evacuation, and drain implantation) was no higher than the risk for stereotactic procedures. This suggests that the risk of bleeding may be more associated with patient comorbidities, including hypertension, rather than with the procedure performed. Nonetheless, one retrospective study compared the risk of ICH in 178 patients who received MER compared to macroelectrode stimulation and found the risk of ICH following DBS correlated with the number of microelectrode trajectories made, the use of MER rather than macroelectrode stimulation, and hypertension [4]. Two hundred and forty-eight functional neurosurgical procedures were performed in these patients, including pallidotomy (122 patients), thalamotomy (19 patients), and implantation of DBS electrodes in the STN, globus pallidus internus, and VIM nucleus. One hundred and forty-four procedures were performed with macroelectrode stimulation, compared to 104 procedures performed with MER. Five cases of hemorrhage $(2.02 \%)$ were observed. The ICH incidence was $2.9 \%$ in patients who received MER compared to $1.4 \%$ in patients in whom MER was not used, although this difference was not significant ( $\mathrm{p}=0.65$ ). ICH occurred in $10.7 \%$ of $\mathrm{pa}-$ tients with a history of hypertension and $0.9 \%$ of those who were nonhypertensive $(\mathrm{p}=0.011)$. The mean maximum intraoperative blood pressure was $157 / 83$ in hypertensive patients who experienced hem- orrhage and 158/85 in nonhypertensive patients who experienced hemorrhage. There was a higher incidence of ICH in patients with a history of hypertension who received MER ( $p=0.034)$ compared with nonhypertensive patients who had not undergone MER. There was an also an increased number of electrode passes through the parenchyma when MER was used ( $\mathrm{p}=0.0001)$, and a positive trend between ICH and multiple passes.

It is possible that the patient presented in this case had a coincidental spontaneous thalamic hypertensive ICH. However, this seems unlikely because the patient's hypertension was well controlled prior to and after surgery, with only one moderately elevated $\mathrm{BP}$ reading following the $\mathrm{ICH}$. Aspirin (81 mg/day) was restarted $61 / 2$ weeks after the surgery, well within the timeframe that is considered safe. The patient was also taking melatonin $(3 \mathrm{mg}$ QHS) which has been noted in 6 case reports to increase the risk of blood clotting abnormalities when taken with warfarin [5]. However, all of these patients demonstrated abnormalities in PTT, whereas PTT was normal in our patient. The interaction with other anticoagulants such as aspirin is not known. It is possible that subclinical damage to cerebral blood vessels from the DBS procedure, coupled with risk factors for an ICH including hypertension and aspirin use led to the delayed hemorrhage. This case raises the question of whether hypertensive patients who have received DBS (with or without MER and multiple electrode passes) should have blood pressures more carefully monitored in the weeks following surgery. It also raises the question of whether the reinstitution of aspirin contributes to the risk and when is the appropriate time to reinitiate its use. Further surveillance of patients who undergo DBS is warranted to identify those who experience a delayed hemorrhage, as well as to identify risk factors for delayed ICH.

\section{References}

1 Fahn SE, Tolosa E, Marin C: Clinical rating scale for tremor; in Jankovic J, Tolosa E (eds): Parkinson's Disease and Movement Disorders, ed 2. Baltimore, Williams and Wilkins, 1988, pp 225-234.

2 Umemura A, Jaggi JL, Hurtig HI, Siderowf AD, Colcher A, Stern MB, Baltuch GH: Deep brain stimulation for movement disorders: morbidity and mortality in 109 patients. J Neurosurg 2003;98:779-784.

3 Binder DK, Rau GM, Starr PA: Risk factors for hemorrhage during microelectrodeguided deep brain stimulator implantation for movement disorders. Neurosurgery 2005;56:722-732.

4 Gorgulho A, De Salles AA, Frighetto L, Behnke E: Incidence of hemorrhage associated with electrophysiological studies performed using macroelectrodes and microelectrodes in functional neurosurgery. J Neurosurg 2005;102:888-896.

5 Herxheimer A, Petrie KJ: Melatonin for the prevention and treatment of jet lag. Cochrane Database Syst Rev 2002;CD001520.

6 Favre J, Taha JM, Burchiel KJ: An analysis of the respective risks of hematoma formation in 361 consecutive morphological and functional stereotactic procedures. Neurosurgery 2002;50:48-56. 